\title{
TWO-BAND ANTENNAS OF MICROWAVE RANGE OF WAVES
}

\author{
V.V. Ovsyanikov, D.M. Svynarenko, O.R. Beznosova*, \\ L.Z. Tsypko \\ Oles Honchar Dnipro National University, Dnipro, Ukraine \\ *e-mail: thalia@i.ua
}

\begin{abstract}
The article presents the results of theoretical and experimental studies of the current distribution, the values and coordinates of the load connection points, as well as the radiation patterns for two-band linear microwave antennas with quarter-wave two-wire shunts of the upper range and concentrated reactive loads. Such antennas are promising for use in two-band duplex mobile satellite systems for receiving and transmitting data, for example, GLOBALSTAR, IRIDIUM, ELLIPSO, GPS and others. Theoretical studies are carried out by the method of integral equation. It is found that with a constant length of a quarter-wave shunt and different sizes of the antenna, the antenna can acquire or lose the property of two frequencies. A method for eliminating this negative phenomenon is proposed.

Keywords: two-band antenna with shunts, current distribution over the antenna, load values and coordinates of their inclusion in the antenna, the radiation patterns of antenna, inductive load.
\end{abstract}

Received 11.09.2019; Received in revised form 12.10.2019; Accepted 15.10.2019

\section{Introduction}

The development of modern facilities of telecommunications, radiolocation, radio navigation, aerospace systems and other industries presents requirements on expanding the frequency range of operation to linear wire antennas and arrays based on them. Two- and multi-frequency antennas are inherently in providing simultaneous transmission or reception of radio waves at several fixed frequencies or frequency ranges. Wherein it is necessary to improve the input parameters, namely the active component of the input resistance $\left(R_{\mathrm{in}}\right)$, close to the wave impedance of the supply feeder, and reactive $\left(Z_{\text {in }}\right)$, equal or close to zero at the given frequencies (ranges) while providing at these frequencies the necessary radiation patterns. Such antennas are in demand for operation in well-known duplex mobile terrestrial and satellite duplex radio communication and information transmission systems, for example, GSM, VODAFONE, LIFE CELL, GLOBALSTAR, IRIDIUM, ELLIPSO, GPS and others. The urgent task of telecommunications is the use of such antennas and antenna arrays in a microstrip design.

In paper [1], a two-frequency symmetric vibrator antenna with so-called loads - "traps" in the form of concentrated oscillating $L C$ circuits connected in series to the antenna branches was considered. The shoulder length of this antenna is $d=0.25 \lambda_{\text {low }}=0.50 \lambda_{\text {up }}$, where $\lambda_{\text {low }}$ and $\lambda_{u p}$ are the wavelengths of the lower and upper operating frequencies. However, such antennas have a reduced efficiency due to Joule losses in their $L C$ circuits and are currently not relevant, since other alternative options with higher values of efficiency in the microwave range are known. These are multi- and two-frequency antennas with resonant coaxial or twowire shunts [2-8] and others.

In well-known works on such multi-frequency and dual-frequency antennas, insufficient information has been published on the analysis of the current distribution on them, input impedance, standing wave voltage coefficient (VSWR), radiation patterns (RP), and optimization of the main characteristics of such antennas. These issues and ways to improve two-band antennas with two-wire shunts are discussed in this article.

\section{Formulation and designing of the problem}

Taking into account the publications [4-8], we carry out a study of the provisions and methods for improving dual-frequency antennas, similar to those shown in Fig. 1. 


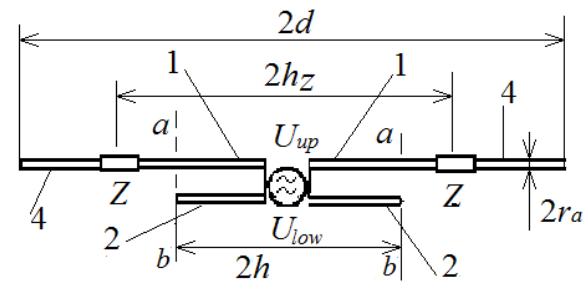

Fig. 1. Two-band antenna $(1,4)$ with linear two-wire shunts 2 , where $Z$ - reactive loads.

The values of the current distribution calculated by the method of integral equation $[8,9]$ on conductors 1,4 with a total length $d$ and conductors 2 of length $h$ (Fig. 1) with an even ratio of compatible frequencies in the absence of loads 4 in conductors $(Z=0)$ are presented graphically in Fig. 2. Here $f_{u p}$ and $f_{\text {low }}$ are the frequencies of the upper and lower wavelengths.

It can be seen from this figure that the amplitude of the frequency $f_{u p}$ current on the protruding part 4 of the conductor $(d-h)$ is much smaller than the amplitude of the current on its other part $(h)$, that is, the effect of "cutting off" the frequency current $f_{u p}$ in section $a-b$ is observed. The calculated value of the current on the protruding part $(d-h)$ compared to its value on the shunt $h$ is weakened to approximately $40 \mathrm{~dB}$.

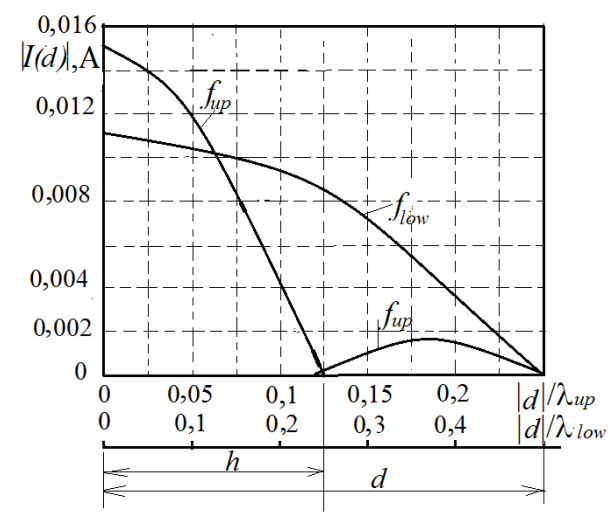

Fig. 2. Distribution of current amplitude on two frequency antenna with working frequency ratio $f_{\text {up }} / f_{\text {low }}=2 / 1$

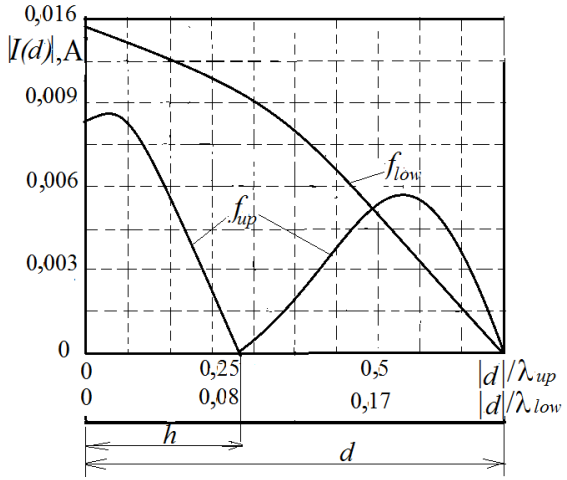

Fig. 3. Distribution of current amplitude on two vibrator antenna with a working frequency ratio $f_{\text {up }} / f_{\text {low }}=3 / 1$

Thus, the frequency current $f_{u p}$, due to the effect of a quarter-wave line shorted in the center of the antenna, encounters a large resistance in section $a-b$ of the two-wire shunt and attenuates significantly on the protruding part 4. In this embodiment, the antenna is operable simultaneously at frequencies $f_{u p}$ and $f_{\text {low }}$, having the usual two-lobe pattern in the form "Eight" at both frequencies, similar to a half-wave vibrator.

In another case (Fig. 3), with an odd ratio of operating frequencies $f_{\text {up }} / f_{\text {low }}=3 / 1$, the antenna vibrator 1 and the protruding part $(d-h)$ (Fig. 1) are intensively excited by the frequency current $f_{u p}$.

This negative effect is a consequence of the fact that the frequency current $f_{u p}$ without obstacles passes to the long vibrator through "opening" the quarter-wave length of the line in section $a-b$, excites the protruding part $(d-h)$ as on a conventional vibrator 
antenna without the shunt. In this case, this antenna acquires the characteristics of a conventional vibrator at a frequency $f_{u p}$.

The property discovered and described above concerning the studied multifrequency antenna with shunts at a frequency ratio $f_{\text {up }} / f_{\text {low }}=3 / 1$ is of considerable scientific and technical interest. It can be explained by the absence of the potential difference at the frequency $f_{u p}$ between the conductors 1 and 2 of the antenna in section $a-b$ of the "opening" of the shunt (Fig. 1), which prevents the locking action of this opening of the quarter-wave line $1-2$.

Considering that in order to ensure the maximum potential difference in section $a-$ $b$, the length of the protruding part 4 should be equal to an odd number of quarters of a short wave $f_{u p}$, the direction of improvement of such antennas should be focused on obtaining in the "openings" of the shunts the sequential resonance mode of the protruding parts $(d-h)$ of the vibrators at a frequency $f_{u p}$ (if we conditionally consider them as individual whip antennas). In this case, the total length of the antenna $2 d$ must be such that a serial resonance mode is created in it also at the frequency $f_{\text {low }}$.

If you implement these two conditions, you can get an antenna operating at randomly spaced two or more frequencies. This mode can be achieved by changing the physical or electrical length of the protruding parts of the vibrators 4 to the values $(d-h)=$ $m 0.25 \lambda_{u p}$ or $(d-h)<0.25 \lambda_{u p}$ where $m=1,3,5, \ldots$, controlling and providing at the same time the resonant size $d / \lambda_{\text {low }} \approx 0.25$.

The indicated electrical length of the protruding parts 4 is proposed to be obtained by sequentially incorporating concentrated or distributed reactive loads $Z$ (Fig. 1) of inductive or capacitive type into their gaps. In this case, the variable adjustable parameters should be the magnitude of the reactive load $Z$, the points of its inclusion $\pm h_{Z}$ and the electric length of the protruding part $(d-h)$. Obviously, the use of inductive loads $(Z)$ in comparison with capacitive loads allows you to slightly reduce the actual length of the antenna, which is a positive factor.

Since the potential of a short quarter-wave rod ' $h$ ' at point ' $b$ ' (Fig. 1) at the frequency $f_{u p}$ is in any case maximum, in order to obtain the maximum potential difference in section $a-b$, the switched-on reactants $Z$ must provide minimum or zero potential at point 'a' of vibrator 1 .

The expression for the value of the inductance of the load resistance $Z$ satisfying this condition, taking into account the theory of the equivalent long line and works [3-8], has the view

$$
L_{r e .1}=\frac{W_{1}}{4 \pi f_{u p}}\left\{\operatorname{ctg}\left[\eta_{(d-h)} \frac{2 \pi}{\lambda_{u p}}\left(d-h_{L}\right)\right]-\operatorname{tg}\left[\eta_{(d-h)} \frac{2 \pi}{\lambda_{u p}}\left(h_{L}-h\right)\right]\right\},
$$

where:

protruding rod 4 wave impedance

$$
W_{1}=120\left[2,3 \lg \frac{d-h}{r_{a}}-1\right]
$$


protrusion shortening factor

$$
\eta_{(d-h)}=1+\left[4,6 \lg \frac{d-h}{r_{a}}\right]^{-1} .
$$

In addition, consistent resonance of antenna (Fig. 1) must be ensured at the frequency $f_{\text {low }}$, and the reactive component of the input resistance at this frequency should vanish or be close to it. The expression for determining the inductance $L_{r e z, 2}$ values that provide this mode, we write approximately as follows

$$
L_{\text {rez } .2}=\frac{W_{2}}{4 \pi f_{\text {low }}}\left\{\operatorname{ctg}\left[\eta_{d} \frac{2 \pi}{\lambda_{\text {low }}}\left(d-h_{L}\right)\right]-\operatorname{tg}\left[\eta_{d} \frac{2 \pi}{\lambda_{\text {low }}} h_{L}\right]\right\} \text {, }
$$

where:

wave impedance of the antenna with shoulder $d$

$$
W_{2}=120\left[2,3 \lg \frac{d}{r_{a}}-1\right],
$$

shortening factor

$$
\eta_{d}=1+\left[4.6 \lg \frac{d}{r_{a}}\right]^{-1}
$$

Thus, from equations (1) and (4) for given operating frequencies and antenna sizes, it is possible to determine the optimal load $Z$ switching points $h_{L}$ with the corresponding value of inductive resistance $\left(L=L_{r e z .1}=L_{r e z .2}\right.$ ), that is, it is possible to model and create a new two-band antenna.

\section{Numerical results}

Consider the results of the calculation and experimental verification of a twofrequency antenna for operation at frequencies with the ratio $f_{\text {up }} / f_{\text {low }}=3 / 1$. It was noted above that conventional antennas with shunts (Fig. 1) without reactive loads $(Z=0)$ do not provide a combination of operating frequencies with odd ratios $f_{\text {up }} / f_{\text {low }}=3,5,7, \ldots$.

As an example, we will examine the possibility of ensuring the operation of a twofrequency antenna in the microwave range with a working frequency ratio $f_{\text {up }} / f_{\text {low }}=3$. Let inductive loads be included in the protruding parts 4 of the antenna (Fig. 1). It is necessary to calculate the antenna with two-wire linear shunts for operation at frequencies $f_{u p}=900 \mathrm{MHz}, f_{\text {low }}=300 \mathrm{MHz}$ with the following initial data: $h=0.25 \lambda_{u p}, d=$ $(0.50 \ldots 0.66) \lambda_{u p}, h_{L}=(0.48 \ldots 0.50) \lambda_{u p}$, supply path wave impedance $\mathrm{w}=50 \mathrm{Ohms}$. Let us determine the dependence of the switched on inductance $L$ and the coordinates of its inclusion on the antenna parameters, including the protruding part $(d-h)$. In this case, we will proceed from the principles set forth in Section 2, performing calculations in accordance with expressions (1) and (4). Moreover, for each value of the length of the vibrator $d$ we get a pair of values $L_{r e z}$ and $h_{L}$.

Shown in Fig. 4, the calculated dependence 1 is calculated by the formula (1) and curve 2 is calculated by the formula (4). The intersection of these curves at point A 
corresponds to the desired pair of values $h_{L}=0,51 \lambda_{\text {up }}$ и $L_{\text {rez }}=0,173 \mu \mathrm{H}$, which is necessary to ensure the two-frequency operation of the antenna.

The radiation patterns (RP) of such an antenna with and without shunts are shown in Fig. 5. At the same time, for an antenna without shunts and $Z$, the RP is multi-lobe and "rugged", like a regular one and a 1.5-wave vibrator have. However, in the presence of shunts and $Z$, the RP becomes uncut, which indicates the positive effect of shunts and $Z$ on the antenna parameters. At its frequency $f_{\text {low }}$, the RP looks like a figure "Eight" - like that of a regular half-wave vibrator.

In Fig. 4 the results of calculations $L_{r e z}$ for various points of inclusion $h_{L}$ of such a load are shown.

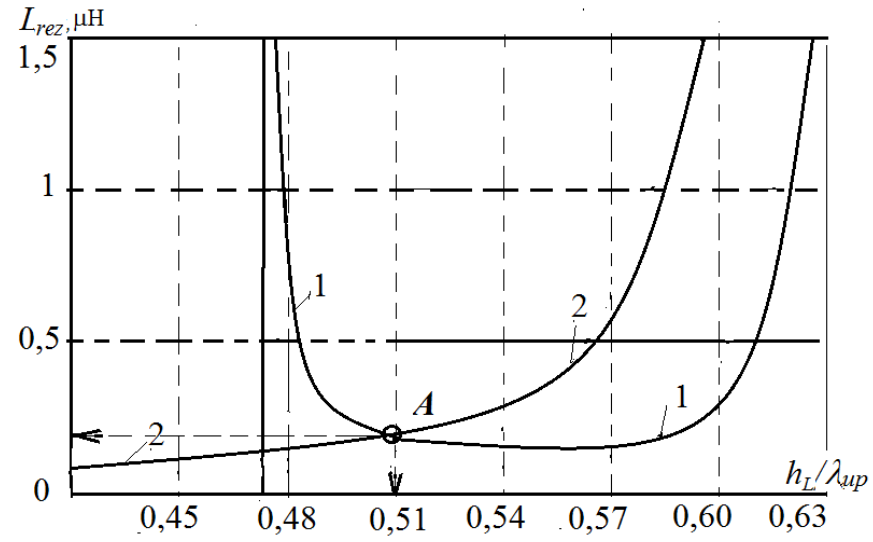

Fig. 4. The calculated dependences $L_{r e z}$ of the loads on the points of their inclusion $h_{L}$ for a given value $d=\mathbf{0 , 6 2}$.

Note that for the initial data given above, each value of the length of the vibrator $d$ and the length of the protruding part $(d-h)$ corresponds to one pair of values $L_{r e z}$ and $h_{L}$, if the electric length of the protruding part does not exceed half the wavelength of the upper frequency $f_{u p}$.

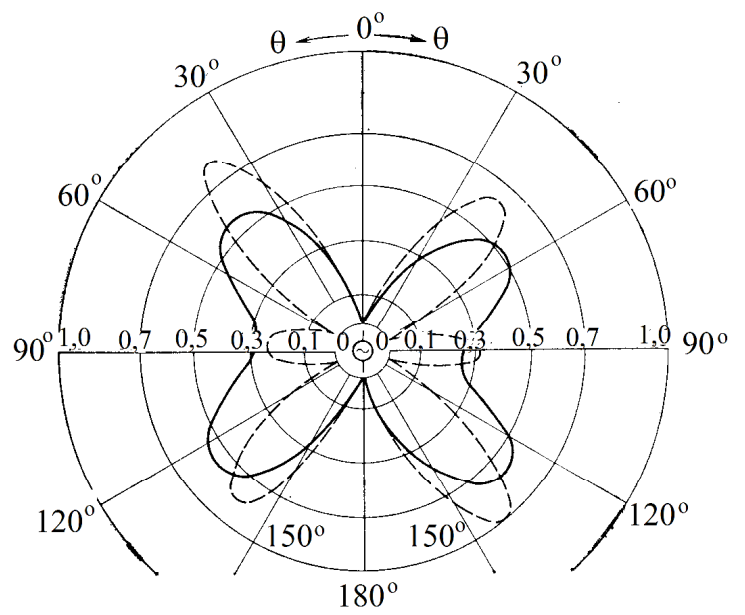

Fig. 5. Experimental radiation patterns of antennas with shunts (-) and without them (---) in the $\mathbf{E}$ plane of the antenna at a frequency $f_{u p}$ with a working frequency ratio $f_{\text {up }} / f_{\text {low }}=3 / 1$ 


\section{Conclusions}

The article proposes a method for constructing a new two-frequency microwave antenna and provides an approximate calculation and experimental analysis using the theory of the equivalent long line. It is shown that to ensure the operation of an electrically short antenna with shunts in two different wavelength ranges, it is necessary to adjust the electric length of the antenna rod that extends beyond the shunt by sequentially including a load in its gap, the value of which must satisfy two conditions: creating the maximum potential difference in the "opening" shunt at the upper operating frequency and ensuring the mode of serial resonance of the antenna at the lower frequency.

As a result of the research, it turned out that with a constant length of the quarterwave shunt mounted on the antenna, and with different sizes of the protruding part of the antenna outside the shunt, the antenna may acquire or lose the property of two ranges. When the length of the rod of the antenna extending beyond the shunt is an odd number of quarters of a short wave of the upper range, the antenna is two-band. If this condition is violated, the antenna loses the property of two ranges. A method for eliminating this negative phenomenon is proposed. It is noted that when creating a real structure considered by two-band antennas, for example, in a microstrip design, it is possible to use more accurate known software environments that take into account the influence of a number of additional details near the antenna.

\section{References}

1. Smith, D.L. The Trap-Loaded Cylindrical Antenna / D.L. Smith // IEEE Trans. 1975.-Vol. AP-23, No. 1.- P. 20 - 27.

2. Pat. 3735413 United States, HO1Q 1/00, 9/16. Multifrequency Antenna System Including an Isolation Section Open Circuit at Both Ends / Oukley McDonald Woodward (USA) Application 19.08.71; Publ. 22.05.73. - 5 p.

3. Patent UA 52711 Ukraina, MKI H01Q 11/10, Antena / Vikt.V. Ovsyanikov, Vl.V. Ovsyanikov (Ukraina), No. 99073884. - Zayavl. 08.07.99. Opubl. 15.01.03, Biul. No. 1. - 2 s.

4. Langley, R. Dual band wearable antenna / R. Langley, Sh. Zhu //Loughborough Antennas \&Propagation Conference - 17-18 March 2008, Loughborough , UK. - P. 14 - 17.

5. Chufarov, M.V. Dual-band microstrip circular polarization antenna for use in GLONASS/GPS systems / M.V. Chufarov, L.A. Lvova, A.V. Babushkin, O.V. Yurchenko // $21^{\text {st }}$ Int Crimean Conference "Microwave \& Telecommunication Technology", 12-16 Sept., 2011, Sevastopol, Ukraine. P. 549 - 550.

6. Galischuk, A.A. Variants of the double frequency GPS antennas / A.A. Galischuk, V.M. Masley, O.L. Ol'shevs'kiy, Y.D. Romanenko, V.V. Zaznobin // XI Internathional Conference on Antenna Theory and Techniques, 24-27 May, 2017, Kyiv, Ukraine. - P. $202-205$.

7. Krjukov, A. The antennas for Mobile Radio Communications / A. Krjukov, V. Ovsyanikov // 31 ${ }^{\mathrm{st}}$ European Microwave Conference - London, 2001 - Vol. 3. - P. 469 - 472.

8. Ovsyanikov, V.V. State of development of vibrator, dielectric and plasma antennas in the context of the historical development of antenna technology / V.V. Ovsyanikov // Radiophysics and Electronics. - Kharkiv: J. IRE NAS of Ukraine. - 2016. - Vol. 7 (21), No. 3. - P. 58 - 73 (in Russian).

9. Ovsyanikov, V.V. Approximate and strict methods for the calculation of rode angle antennas of microwave range / V.V. Ovsyanikov, D.M. Svynarenko, E.R. Beznosova, L.Z. Tsipko // Journ. of Phys. and Electronics (ISSN 2616-868). - 2018. Vol. 26 (1). -. P. $83-88$. 